\title{
Analysis of a case with disappearance of the primary gastrointestinal stromal tumor and progressive liver metastases under long-term treatment with tyrosine kinase inhibitors
}

\author{
Silke Cameron · Theodoros Savvoukidis • \\ Thomas Armbrust • Florian Haller • \\ Julia Kitz · László Füzesi · Giuliano Ramadori
}

Received: 1 February 2009/ Accepted: 26 February 2009/Published online: 18 March 2009

(C) Humana Press Inc. 2009

\begin{abstract}
The response of gastrointestinal stromal tumors (GISTs) to tyrosine kinase receptor inhibitors (TKR-I) has been a breakthrough for small molecular therapy. We report here on the very different long-term outcome of a synchronous metastatic GIST with complete remission of the primary tumor and progressive liver metastases under TKR-I therapy. In 2003, a 52-year-old patient was diagnosed with gastric GIST and synchronous multiple liver metastases. Therapy with imatinib, $400 \mathrm{mg}$ daily, was started immediately. Fifteen months later, the primary was no longer detectable by endoscopy. In 2006, progression of the liver metastases was observed. Mutation analysis of the initial biopsy specimen from the primary, as well as the biopsy from the three main liver metastases after 3 years of imatinib treatment, revealed the common KIT exon 11 deletion (W557_K558del) in all tumor samples. Two of the metastases had a separate secondary mutation in KIT exon 14 and 17, respectively, while the largest cystic metastatic lesion had no other mutation. Imatinib was then increased to a daily dose of $800 \mathrm{mg}$, and in April 2007 the treatment was changed to sunitinib. Fifty-two months after initial diagnosis, the patient died of liver failure. At no time point, relapse of the gastric primary tumor was observed. Whilst
\end{abstract}

Dedicated to Prof. Dr. Dr. h.c. Karl-Hermann Meyer zum Büschenfelde on the occasion of his 80th birthday.

S. Cameron · T. Savvoukidis · T. Armbrust · G. Ramadori ( $\square)$ Department of Gastroenterology and Endocrinology,

University Clinic of Göttingen, Robert-Koch-Strasse 40, Göttingen D-37099, Germany

e-mail: gramado@med.uni-goettingen.de

F. Haller · J. Kitz · L. Füzesi

Department of Gastroenteropathology, University Clinic of the

Georg August University, Robert-Koch-Strasse 40,

Göttingen D-37099, Germany
TKR-Is are commonly very effective in treating GISTs, the present case illustrates their varying effects regarding the clinical behavior and genetic variations within different tumors of the same patient after long-term treatment.

Keywords Gastrointestinal stromal tumor (GIST) . Secondary mutations $\cdot$ Mutation analysis .

Tyrosine kinase receptor inhibitor (TKR-I) .

Treatment response of primary GIST versus metastases

\section{Introduction}

Gastrointestinal stromal tumors (GISTs) are the most common mesenchymal tumors of the gastrointestinal tract [1]. The incidence of GISTs is approximately 20 cases per million [2], the stomach being the most frequent site of origin. Activating mutations of the proto-oncogene receptor tyrosine kinase KIT [3] or the structurally related platelet-derived growth factor receptor $\alpha$ (PDGFRA) [4] are regarded as central pathogenic events in the tumorigenesis of GISTs [5].

In a study of 200 patients with malignant GIST, approximately $50 \%$ were found to be present with synchronous metastases [6]. The liver and the peritoneal cavity are the most frequent targets of GIST metastasis. Surgical resection, when practicable, is the primary therapy of choice and, for a long time, has been the unique therapeutic approach. Although complete resection can be achieved in $86 \%$ of the patients, relapse of high-risk GIST after R0resection is common within the first 2 years after operation [6]. The overall 5-year survival rate after resection without further adjuvant treatment lies between 34 and $42 \%$, due to the local recurrence or metastatic spread [7, 8].

The use of imatinib, a small bioactive molecule inhibiting the tyrosine kinase receptor (TKR-I) activity of c-kit 
and PDGFRA has influenced the survival of metastasized or relapsed GIST dramatically [9]. Imatinib soon became the primary treatment modality of advanced (metastasized or unresectable) GIST $[9,10]$. In special cases, imatinib treatment may render non-resectable tumors resectable [11]. To our knowledge, we here report for the first time on the long-term eradication of a primary gastric GIST. As the primary tumor was not resected due to the advanced stage with synchronous liver metastases, we can address the difference in treatment response of the primary GIST and its synchronous metastases to TKR-I therapy.

\section{Case report}

A 52-year-old male patient first presented in December 2003 with persistent back pain which was interpreted to be due to his already known chronic degenerative vertebral spinal disorder. Computed tomography (CT) scan was performed and multiple hypodense hepatic masses were detected (Fig. 1). At that time, the patient had no other symptoms except for the known back pain. The serological tumor markers for epithelial gastrointestinal tumors (CEA, CA 19-9) were all negative. Pathological examination after liver needle biopsy showed positivity for c-kit (CD 117), and a high proliferation index of $70 \% \mathrm{Ki}-67$ (Mib1) positive cells (Fig. 1). Liver metastasis of GIST was diagnosed. Retrospective mutation analysis was not possible, as no additional tumor material was available.

The primary tumor was detected in the subcardial area of the stomach. It was $5 \mathrm{~cm}$ large and protruding into the gastric lumen with central ulceration. Biopsy confirmed the primary GIST (Fig. 1). Retrospective mutation analysis of the primary tumor specimen revealed a six basepair in-frame deletion in exon 11 of KIT (c.1756_1761delTGGAAG), leading to deletion of two amino acids at position 557-558 (p.Trp557_Lys558del). As the tumor showed synchronous liver metastases, therapy with imatinib, $400 \mathrm{mg}$ daily, was started.

In March 2005, after 15 months of pharmacological treatment, the primary tumor of the stomach was endoscopically no longer detectable (Fig. 1, inlay). Until then,
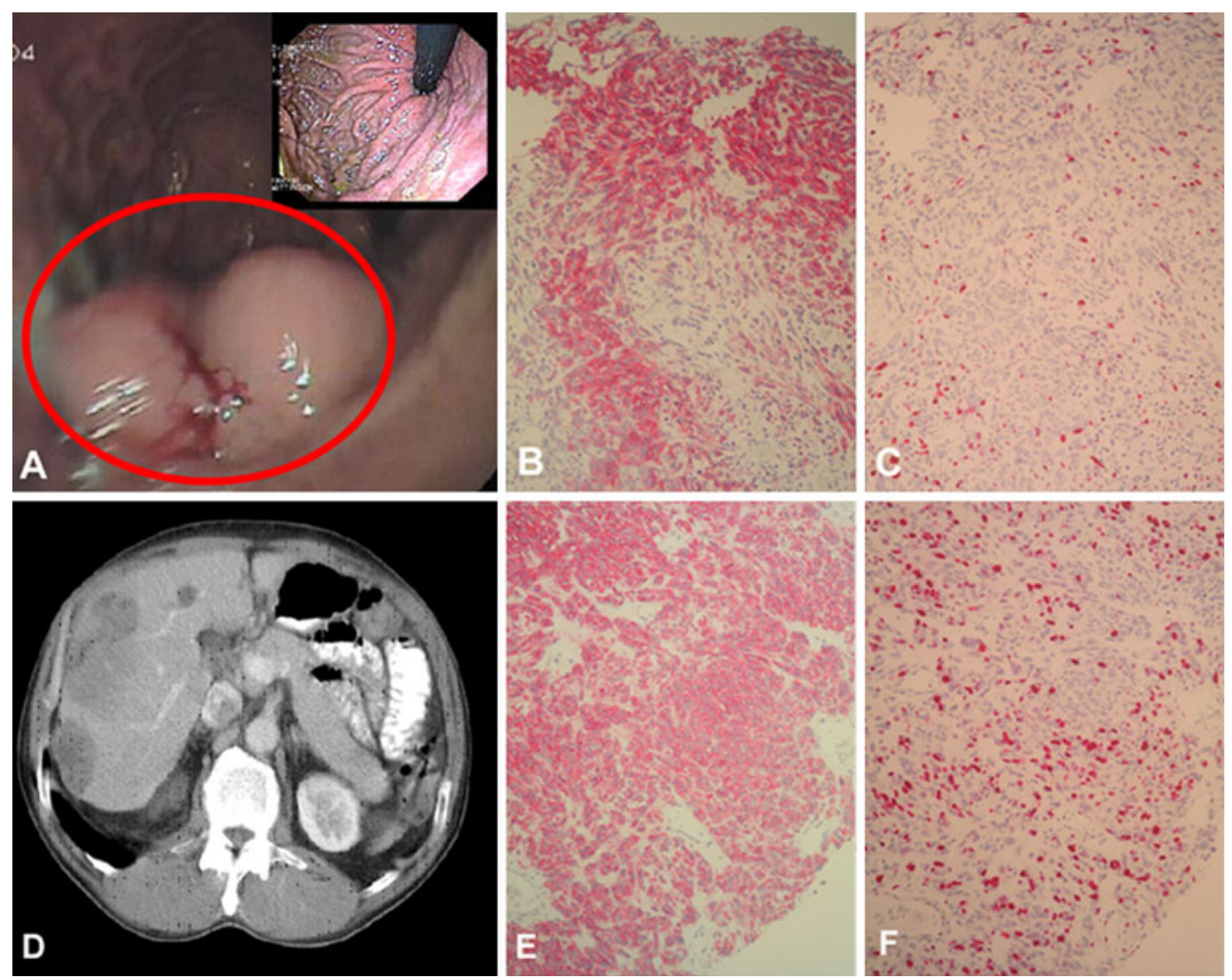

Fig. 1 Gastric GIST (a-c) and synchronous liver metastases $(\mathbf{d}-\mathbf{f})$ at diagnosis. a Gastroscopic image of the $5 \mathrm{~cm}$ large tumor with central ulceration in the subcardial area of the stomach. The inlay shows the subcardial area after imatinib therapy, no tumor can be observed. d CT-slice of the upper abdomen prior to initiating imatinib therapy. Several hypo- to isodense lesions are shown in both liver lobes. b, c and $\mathbf{e}, \mathbf{f}$ Consecutive slides of formalin-fixed and paraffin-embedded tissue sections of the primary. All photographs were taken at an original magnification of $200 \times$; b and e anti-ckit stainig (DAKO, A4502) showing c-kit positivity of the primary and the metastasis, respectively; c and $\mathbf{f}$ anti-MIB1-staining (Ki67 clone MIB1, DAKO M7240) showing a low proliferation index of about $15 \%$ Ki67 positive cells in the primary (c) and of about $70 \%$ in the metastasis (f) 
partial regression of the metastases was observed. At this time, the more anteriorly located solid metastasis (segment V) decreased from about 6 to $3.5 \mathrm{~cm}$. The more distally located solid metastasis (segment VI) showed a slight initial regression from $7 \mathrm{~cm}$ to about $4 \mathrm{~cm}$. And the largest liver metastasis (segment VII) showed cystic regression and a size decrease in March 2003 from $7 \mathrm{~cm}$ to $5 \mathrm{~cm}$. However, it has to be noted that differentiation between the metastases in segment VII and V was difficult.

The patient was first referred to our hospital in October 2006, after 34 months of imatinib treatment ( $400 \mathrm{mg} / \mathrm{day}$ ). Whilst the primary tumor remained undetectable, CT-scan,
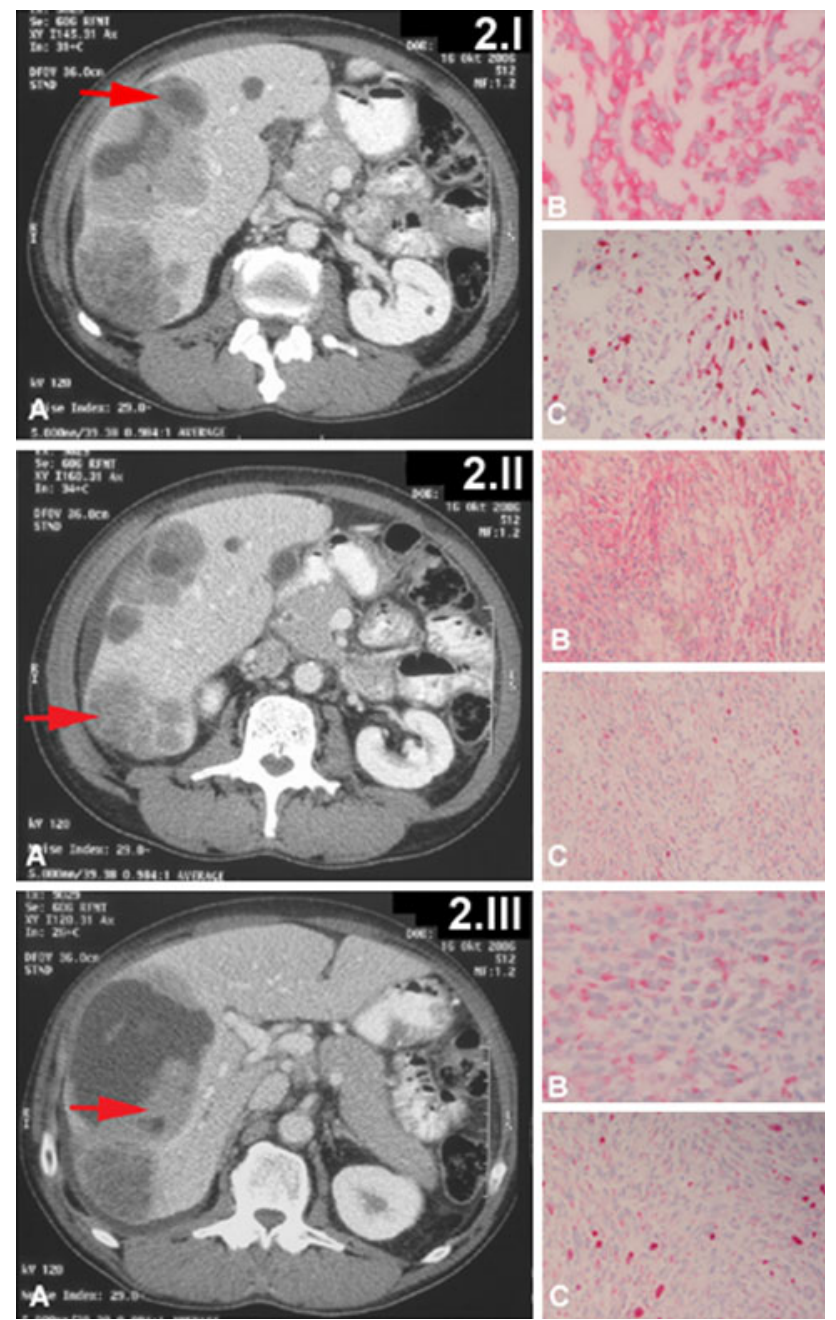

Fig. 2 Progress of the liver metastases after 34 months of imatinib treatment $(400 \mathrm{mg} / \mathrm{d})$. a CT slices of the upper abdomen. b-c show consecutive slides of formalin-fixed and paraffin-embedded tissue sections of the different liver lesions: $2 . \mathrm{I}: 3.5 \mathrm{~cm}$ large metastasis (segment V); $2 . \mathrm{II}: 7 \mathrm{~cm}$ large metastasis (segment VI); $2 . \mathrm{III}: 10 \mathrm{~cm}$ large cystic metastasis (segment VII). All photographs were taken at an original magnification of $200 \times$; b anti-ckit stainig (DAKO, A4502) with strong to weak c-kit positivity; c anti-MIB1-staining (Ki67 clone MIB1, DAKO M7240) showing a proliferation index of 2.I: $30 \%$; 2.II: $5 \%$; and 3.III of $20 \% \mathrm{Ki} 67$ positive cells
FDG-PET, and ultrasound revealed an enlargement of the hepatic metastases (Fig. 2).

At this time point, fine needle biopsy of all three metastases was performed (Fig. 2). As the biopsy specimen were too small to be evaluated for mitotic count in 50 high power fields (HPFs), the proliferation rate was assessed immunohistochemically by Mib1 (Ki67 clone Mib1) staining. The tissue fragments were analysed for mutation of KIT exon 9, 11, 13, 14, 15, 16, 17, and PDGFRA exon 12,14 , and 18 as described previously [12].

The following results were obtained:

a) Segment V: The metastasis had remained constant at $3.5 \mathrm{~cm}$ under imatinib therapy. The proliferation rate, however, was high with $30 \%$. The initial KIT exon 11 deletion was found as well as a further point mutation at position 2096 in KIT exon 14 (c.2096C > T), leading to a single amino acid change at position 670 (p.Thr670Ile).

b) Segment VI: The metastasis had regained its original size of $7 \mathrm{~cm}$, whilst the proliferation rate remained low at $5 \%$. The metastasis was found to have the primary deletion in KIT exon 11 and a further point mutation at position 2554 in $K I T$ exon 17 (c.2554T $>$ G), leading to a single amino acid change at position 823 (p.Tyr823Asp).

c) Segment VII: The cystic metastasis showed a size increase from 5 to $10 \mathrm{~cm}$. The proliferation rate within the tumor material from the border of the cystic lesion was $20 \%$. Mutation analysis showed the initial primary mutation in KIT exon 11.

Both additional point mutations in exon 14 and 17 were found only in the metastases and under imatinib treatment. At this time point (after 34 months), the imatinib dose was increased to $800 \mathrm{mg}$ daily.

Four months later (February 2007) a CT-scan detected a further size increase of the $10 \mathrm{~cm}$ large cystic metastasis (segment VII) to $14 \mathrm{~cm}$ (Fig. 3). An extrahepatic focus was not detected, neither in the abdominal CT-scan, nor in FDG-PET.

Meanwhile the patient developed strong abdominal pain, ascites, and right pleural effusion, which was related to partial compression of the inferior vena cava, as well as to a thrombosis of the right portal vein.

In April 2007, about 40 months after initiation of imatinib therapy, the treatment was changed to sunitinib (50 $\mathrm{mg}$ per day) in the attempt to reduce the size of the metastases. As the liver values were elevated at this stage, the treatment was given on a 2 weeks on, instead of a 4 weeks on schedule. This treatment had to be further reduced to $25 \mathrm{mg}$ as a phototoxic skin reaction occurred. Under sunitinib therapy, the metastasis in segment $\mathrm{V}$ became cystic, whilst the metastasis in segment VI started to grow (Fig. 3). The largest metastasis (segment VII) showed further cystic regression. 
Fig. 3 Comparison of the different liver metastases after increased dosage of imatinib and after change to sunitinib therapy. The images show CT-scans of a caudal and cranial transverse section of the liver at two different time points. The metastasis harboring an additional KIT exon 14 mutation (segment V) became cystic under sunitinib treatment. The metastasis with an additional KIT exon 17 mutation (segment VI) remained stable under imatinib and increased in size under sunitinib treatment. The dominant metastasis in the right liver lobe became cystic under imatinib and further sunitinib treatment (KIT exon 11 mutation, lower panel)
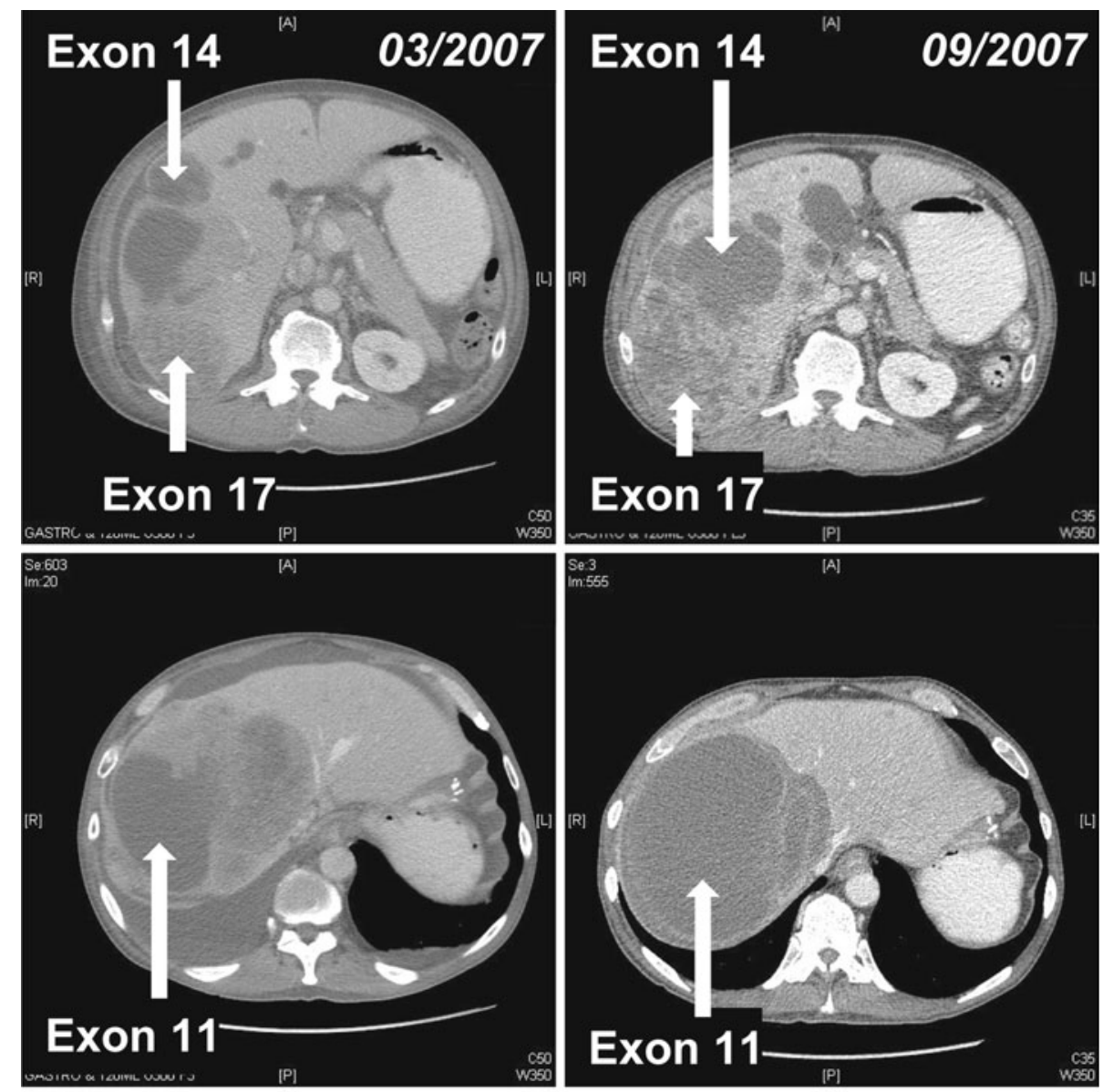

Imatinib $800 \mathrm{mg}$ $(10 / 06-04 / 07)$
Sunitinib 50-25-50 mg (04/2007-10/2007)
With general disease progression, including the formation of new hepatic lesions especially in the left liver lobe, the treatment was changed back to imatinib in September 2007. One month later, explorative laparotomy was performed in the attempt to resect the largest tumor mass. Another 5 months later, the patient died of liver and renal failure. At no time point, relapse of the gastric primary was observed.

\section{Discussion}

We have described the very different behavior of each, the primary gastric GIST and three synchronous liver metastases under TKR-I therapy.

The $5 \mathrm{~cm}$ large primary gastric GIST was not detectable endoscopically after 15 months of imatinib therapy and did not recur over the observation period of overall 52 months. It had a relatively common KIT exon 11 in-frame deletion, leading to deletion of amino acids 557-558, which are part of the juxtamembrane domain. It is known that GISTs with gain-of-function mutations in KIT exon 11 are most responsive to imatinib treatment [13]. To our knowledge, complete clinical response of the primary over such a long observation time has not previously been reported. In a review of 10 cases undergoing neoadjuvant imatinib treatment, primary tumor size decreased to about $20-70 \%$ of the initial size over a treatment duration of in the mean 5 months [12]. The most common mutations were also reported during KIT exon 11 mutations [12, 14]. However, there is a discussion about GISTs which, after so-called complete pathological response (with the absence of tumor cells and the formation of a hyaline tissue), show relapse at a distant site [15-18]. In our case, however, with advanced disease, resection of the primary was not performed and therapy with TKR-I was never stopped. Instead, the metastases were the issue.

Mutation analysis of the liver metastases showed the primary KIT exon 11 mutation in each of the three metastases. No secondary mutation was found in the largest 
metastasis in segment VII which showed cystic regression over the course of therapy. A challenge of this regression pattern was an increase in volume of the lesion which led to a compression of the inferior vena cava and to thrombosis of the right portal vein.

Separate typical secondary mutations were found in $K I T$ exons 17 (liver segment VI) and 14 (segment V) under imatinib therapy. Whilst the latter became cystic under sunitinib treatment, the metastasis in segment VI did not show regression under sunitinib treatment.

Secondary mutations are a challenge for successful long-term therapy in GISTs. The best clinical benefit (i.e., partial response or stable disease) with sunitinib has been observed for the most common primary GIST genotypes: KIT exon 9 (dimerization domain), and KIT exon 11 (juxtamembrane domain) [19]. Instead, secondary mutations during long-term imatinib therapy occur in KIT exon 13-17 [19-21]. Imatinib resistance in these regions is usually confered by secondary point mutations which are located in the drug/adenosine triphosphate binding pocket of the receptor which is encoded by exon 13 and 14 or in the activation loop, encoded by exon 17 (tyrosine kinase domain 2) [19]. These point mutations lead to single amino acid changes at specific positions and inhibit successful binding of imatinib. For exon 17 mutations, clinical responses to imatinib have been observed, even though this mutation occurs under imatinib treatment [13], so that different acting mechanisms for imatinib cannot be excluded. KIT exon 13-17 mutations are rare in primary GISTs without imatinib treatment $(<2 \%)$ [22], while they are found in high frequency in advanced GISTs with secondary resistance after long-term imatinib treatment [23].

For the KIT exon 14 mutation T670I, recent studies have reported that - in contrast to imatinib — sunitinib successfully inhibits the kinase activity of mutated KIT [19]. With sunitinib, progression-free and overall survival for patients with secondary KIT exon 14 mutations are longer than for those with KIT exon 17 mutations [19].

Our data are in agreement with these results. In our case, the metastasis with the additional KIT exon 17 mutation showed initial stability under imatinib with a low proliferation rate $(5 \%)$ and did not respond to sunitinib treatment (Fig. 3). The metastatic lesion with the additional KIT exon 14 mutation displayed a high proliferation rate of $30 \%$. It became cystic under sunitinib. We would like to point out that the metastasis with the solely primary KIT exon 11 mutation which had a proliferation rate of $20 \%$ also became cystic.

We have to ask, whether combination of imatinib, sunitinib and, or other targeted therapies is possible and to which extent. To help answer this question, additional to prognostic factors for the classification of GIST biology such as tumor size, location, and mitosis [24-26] which have been found whilst analysing untreated primary GISTs, predictive factors such as biomarkers need to be identified, maybe for every metastasis. This would help an even more individual treatment approach. To date, only mutation analysis has gained general acceptance as a useful predicting factor for therapeutic outcome [27].

More reports are needed which observe the individual behavior of the different metastases in terms of progression and regression pattern under therapy. To date, four different progression patterns have been defined: focal progression, general progression, development of new cystic lesions, and new solid lesions [28]. As for regression patterns no such classification exists. And to our understanding, cystic lesions can develop in rapidly growing tumors, however, they are usually a sign of tumor regression. Our observations underscore the heterogeneity of the behavior of the different lesions under tyrosine kinase inhibitor treatment [29].

In summary, we could show that under therapy with TKR-I, the primary behaves different from the metastases (even if it harbors the same mutation) and that the different metastases behave different from each other (with the same primary mutation but different secondary mutations). Hence, a difficulty arises when mutations with different response rates are found within the same patient. If curative surgery cannot be performed, the adequate time point to change from imatinib to sunitinib treatment remains to be clarified [19]. We learnt that sunitinib should not be employed too late in the course of the disease when liver function and general condition have declined. On the other hand, metastases which are under control with imatinib, may not respond to sunitinib.

Acknowledgment We are thankful to all our colleagues who participated in the treatment of the patient.

\section{References}

1. Miettinen M, Lasotta J. Gastrointestinal stromal tumorsdefinition, clinical, histological, immunohistochemical, and molecular genetic features and differential diagnosis. Virchows Arch. 2001;438:1-12. doi:10.1007/s004280000338.

2. Kindblom LG, et al. Incidence, prevalence, phenotype and biologic spectrum of gastrointestinal stromal cell tumors (GIST) - A population based study of 600 cases. Ann Oncol. 2002;13(Suppl 5):157. doi:10.1093/annonc/mdf012.

3. Hirota S, et al. Gain-of-function mutations of c-Kit in human gastrointestinal stromal tumors. Science. 1998;279:577-80. doi: 10.1126/science.279.5350.577.

4. Heinrich MC, et al. PDGFRA activating mutations in gastrointestinal stromal tumors. Science. 2003;299:708-10. doi:10.1126/ science. 1079666.

5. Hirota S, Isozaki K. Pathology of gastrointestinal stromal tumors. Pathol Int. 2006;56:1-9. doi:10.1111/j.1440-1827.2006.02036.x.

6. DeMatteo RP, et al. Two hundred gastrointestinal stromal tumors: recurrence patterns and prognostic factors for survival. Ann Surg. 2000;231:51-8. doi:10.1097/00000658-200001000-00008. 
7. Pidhorecky I, Cheney RT, Kraybill WG, Gibbs JF. Gastrointestinal stromal tumors: current diagnosis, biologic behavior, and management. Ann Surg Oncol. 2000;7:705-12. doi:10.1007/ s10434-000-0705-6.

8. Pierie JP, et al. The effect of surgery and grade on outcome of gastrointestinal stromal tumors. Arch Surg. 2001;136:383-9. doi: 10.1001/archsurg.136.4.383.

9. DeMatteo RP, Heinrich MC, El-Rifai WM, Demetri G. Clinical management of gastrointestinal stromal tumors: before and after STI-571. Hum Pathol. 2002;33:466-77. doi:10.1053/hupa.2002. 124122.

10. Joensuu $\mathrm{H}$, et al. Effect of the tyrosine kinase inhibitor STI571 in a patient with a metastatic gastrointestinal stromal tumor. N Engl J Med. 2001;344:1052-6. doi:10.1056/NEJM200104053441404.

11. DeMatteo RP, et al. Results of tyrosine kinase inhibitor therapy followed by surgical resection for metastatic gastrointestinal stromal tumor. Ann Surg. 2007;245:347-52. doi:10.1097/01.sla. 0000236630.93587.59.

12. Haller F, et al. Surgical management after neoadjuvant imatinib therapy in gastrointestinal stromal tumours (GISTs) with respect to imatinib resistance caused by secondary KIT Mutations. Ann Surg Oncol. 2006;14:526-32. doi:10.1245/s10434-006-9228-0.

13. Joensuu H. Gastrointestinal stromal tumor (GIST). Ann Oncol. 2006;17(Suppl 10):280-6. doi:10.1093/annonc/mdl274.

14. Bümming P, et al. Neoadjuvant, adjuvant and palliative treatment of gastrointestinal stromal tumours (GIST) with imatinib: a centre-based study of 17 patients. Br J Cancer. 2003;89:460-4. doi:10.1038/sj.bjc.6600965.

15. Salazar M, et al. First report of a complete pathological response of a pelvic GIST treated with imatinib as neoadjuvant therapy. GUT. 2006;55:585-6. doi:10.1136/gut.2005.086744.

16. Bauer S, Lang H, Schütte J, Hartman JT. Complete remission with imatinib in metastatic gastrointestinal stromal tumors. J Clin Oncol. 2005;23:6800-1. doi:10.1200/JCO.2005.02.1063.

17. Chacon M, Roca E, Huertas E, Sanchez Loria F, Domenechini E. Pathologic complete remission of metastatic gastrointestinal stromal tumor after imatinib mesylate. J Clin Oncol. 2005; 23:1580-2. doi:10.1200/JCO.2005.03.194.
18. Bauer $\mathrm{S}$, et al. Resection of residual disease in patients with metastatic gastrointestinal stromal tumors responding to treatment with imatinib. Int $\mathrm{J}$ Cancer. 2005;117:316-25. doi: 10.1002/ijc. 21164 .

19. Heinrich MC, et al. Primary and secondary kinase genotypes correlate with the biological and clinical activity of sunitinib in imatinib-resistant gastrointestinal stromal tumor. J Clin Oncol. 2008;26:5352-9. doi:10.1200/JCO.2007.15.7461.

20. van der Zwan S, DeMatteo RP. Gastrointestinal stromal tumor: 5 years later. Cancer. 2005;104:1781-8. doi:10.1002/cncr.21419.

21. Lim K-H, et al. Molecular analysis of secondary kinase mutations in imatinib-resistant gastrointestinal stromal tumors. Med Oncol. 2008;25:207-13. doi:10.1007/s12032-007-9014-2.

22. Rubin BP. Gastrointestinal stromal tumours: an update. Histopathol. 2006;48:83-96. doi:10.1111/j.1365-2559.2005.02291.x.

23. Heinrich MC, et al. Molecular correlates of imatinib resistance in gastrointestinal stromal tumors. JCO. 2006;24:4764-74. doi: 10.1200/JCO.2006.06.2265.

24. Fletcher CDM, et al. Diagnosis of gastrointestinal stromal tumors: a consensus approach. Hum Pathol. 2002;33:459-65. doi: 10.1053/hupa.2002.123545.

25. Miettinen M, El-Rifai W, Sobin LH, Lasota J. Evaluation of malignancy and prognosis of gastrointestinal stromal tumors: a review. Hum Pathol. 2002;33:478-83. doi:10.1053/hupa.2002. 124123.

26. Joensuu H. Risk stratification of patients diagnosed with gastrointestinal stromal tumor. Hum Pathol. 2008;39:1411-9. doi: 10.1016/j.humpath.2008.06.025.

27. Judson IR. Prognosis, imatinib dose, and benefit of sunitinib in GIST: knowing the genotype. J Clin Oncol. 2008;26:5322-5. doi: 10.1200/JCO.2008.17.7725.

28. Ryu M-H, et al. Patterns of progression in gastrointestinal stromal tumor treated with imatinib mesylate. Jpn J Clin Oncol. 2006;36:17-24. doi:10.1093/jjco/hyi212.

29. Liegl B, et al. Heterogeneity of kinase inhibitor resistance mechanisms in GIST. J Pathol. 2008;216:64-74. doi:10.1002/ path.2382. 